\title{
miR-873 inhibits colorectal cancer cell proliferation by targeting TRAF5 and TAB1
}

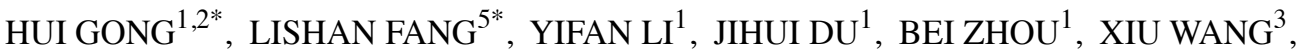 \\ HEKAI ZHOU $^{1}$, LINGLI GAO ${ }^{1}$, KAIXIN WANG ${ }^{4}$ and JUAN ZHANG ${ }^{4}$ \\ ${ }^{1}$ Central Laboratory, ${ }^{2}$ Shenzhen Key Laboratory of Endogenous Infection, ${ }^{3}$ Clinical Laboratory and ${ }^{4}$ Department of Pathology, \\ Shenzhen Nanshan People's Hospital/Affiliated Shenzhen Sixth Hospital of Guangdong Medical University; \\ ${ }^{5}$ Central Laboratory, The Eighth Affiliated Hospital of Sun Yat-sen University, Shenzhen, Guangdong 518033, P.R. China
}

Received June 20, 2017; Accepted December 20, 2017

DOI: $10.3892 / o r .2018 .6199$

\begin{abstract}
MicroRNA-873 (miR-873) has been reported to be dysregulated in a variety of malignancies, however, the biological function and underlying molecular mechanism of miR-873 in colorectal cancer (CRC) remain unclear. In the present study we found that the expression levels of miR-873 were markedly decreased in CRC cell lines and tissues from patients. Statistical analysis revealed that miR-873 expression was inversely correlated with the disease stage of CRC. Kaplan-Meier survival analysis revealed that patients with CRC with lower miR-873 expression had shorter overall survival rates. Additionally, downregulation of miR-873 enhanced the proliferation of CRC cells, while upregulation of $\mathrm{miR}-873$ reduced this proliferation. Furthermore, we found that tumor necrosis factor (TNF) receptor-associated factor 5 (TRAF5) and TGF- $\beta$ activated kinase 1 (MAP3K7) binding protein 1 (TAB1) were direct targets of miR-873 in CRC cells. A luciferase assay revealed that ectopic expression of miR-873 significantly reduced nuclear factor $\kappa \mathrm{B}(\mathrm{NF}-\kappa \mathrm{B})$ luciferase activity, while ectopic expression of miR-873 inhibitor enhanced luciferase activity, suggesting that downregulation of miR-873 can activate NF- $\kappa B$ signaling. Therefore, our findings established a tumor-suppressive role for miR-873 in the inhibition of CRC progression, which may be employed as a novel prognostic marker and as an effective therapeutic target for CRC.
\end{abstract}

\section{Introduction}

Colorectal cancer (CRC), one of the most common malignancies of the gastrointestinal tract, is a major cause of

Correspondence to: Dr Hui Gong, Central Laboratory, Shenzhen Nanshan People's Hospital/Affiliated Shenzhen Sixth Hospital of Guangdong Medical University, 86 Taoyuan Road, Shenzhen, Guangdong 518052, P.R. China

E-mail: gonghui2008ok@163.com

*Contributed equally

Key words: miR-873, TRAF5, TAB1, NF-кB, colorectal cancer, proliferation tumor-associated morbidity and mortality worldwide (1). Its incidence rate continues to rise (2). Over 1 million new cases of CRC are diagnosed globally each year, resulting in about 0.5 million deaths annually $(3,4)$. The poor prognosis for $\mathrm{CRC}$ is mainly attributable to its insidious onset, atypical symptoms, and aggressive malignancy. Most patients with CRC are diagnosed at an advanced stage. Approximately $25 \%$ of patients with CRC present with liver metastases at the time of the initial diagnosis (5). Despite overall advances in the treatment of this disease, the overall cure rate of CRC has not markedly improved, and the overall 5-year survival rate remains at approximately $60 \%$ in Asia (6).

Nuclear factor $\kappa \mathrm{B}(\mathrm{NF}-\kappa \mathrm{B})$ is a family of dimeric transcription factors required to coordinate numerous physiological and pathological processes, such as immunity, inflammation, and tumorigenesis $(7,8)$. Notably, constitutively activated $\mathrm{NF}-\kappa \mathrm{B}$ signaling has been demonstrated to play vital roles in the development and progression of a large array of malignancies, including CRC $(9,10)$. Stimulatory factors, such as tumor necrosis factor- $\alpha$ (TNF- $\alpha)$, interleukin-1 $\beta$ (IL-1 $\beta)$, or pathogen-derived components that include bacterial lipopolysaccharides (LPS), bind to their respective receptors, leading to the rapid recruitment of tumor necrosis factor receptor type 1-associated DEATH domain (TRADD), cellular inhibitor of apoptosis protein 1 (cIAP1), baculoviral IAP repeat-containing protein 3 (cIAP2), and TNF receptor-associated factors (TRAFs), including TRAF1, TRAF2, TRAF3, TRAF5, and TRAF6. As molecular activators for the NF- $\kappa$ B signaling pathway, TRAFs function as E3 ubiquitin ligases that induce the K63 polyubiquitination of receptor-interacting protein 1 (RIP1), resulting in activation of the transforming growth factor-activated kinase-1 (TAK1)/TAB2/3/ TGF- $\beta$ activated kinase 1 (MAP3K7) binding protein 1 (TAB1) complex. During this process, TAB1, TAB2, and TAB3 form a complex with TAK1, which phosphorylates and activates the inhibitor of the NF- $\kappa \mathrm{B}$ kinase (IKK) $-\alpha / \beta / \gamma$ kinase complex, leading to nuclear translocation and activation of $N F-\kappa B$ (11-13). Thus, further investigations into the mechanism of regulation of the $\mathrm{NF}-\kappa \mathrm{B}$ pathway key components, such as TRAF2, TRAF5, TAK1, TAB1, and TAB3, would increase our knowledge of the mechanisms underpinning the constitutive activation of $\mathrm{NF}-\kappa \mathrm{B}$ in cancer. 
MicroRNAs (miRNAs), a class of small non-coding RNAs, function as negative regulators of gene expression by interacting with the $3^{\prime}$ untranslated region (3'-UTR) of their target mRNAs (14). These miRNAs, which are approximately 20-25 nucleotides in length, play important roles in a variety of physiological and pathological processes, such as development, cell proliferation, differentiation, and senescence (15). Previous research has revealed that miRNAs are involved in carcinogenesis via regulation of several key cellular processes, including cell proliferation, apoptosis, migration, invasion, and angiogenesis (16). Numerous miRNAs have been reported to be upregulated or downregulated in various types of cancer, demonstrating their potential roles as oncogenes or tumor suppressors (17-20). The altered expression of miRNAs in cancers suggests that they may serve as potential diagnostic or prognostic biomarkers for cancer (21-23). For instance, in glioma, ovarian cancer, and breast cancer, miR-873 is downregulated and may act as tumor suppressor (24-28). However, miR-873 has been revealed to be upregulated in lung adenocarcinoma, where it promotes tumor cell proliferation and migration (29).

In the present study we revealed that miR-873 was downregulated in CRC and this was correlated with a poor prognosis for patients with CRC. Furthermore, we determined that downregulation of miR-873 enhanced CRC cell proliferation by directly targeting TRAF5 and TAB1, leading to activation of NF- $\kappa B$ signaling. These results demonstrated that miR-873 served as a tumor-suppressive miRNA in the development and progression of CRC.

\section{Materials and methods}

Cells. The CRC cell lines (SW620, SW480, DLD1, HCT116, LoVo, and HT-29) were purchased from the American Type Culture Collection (Manassas, VA, USA). The normal human colon mucosal epithelial cell line (NCM460) and the CRC cell line (KM12) were purchased from the BeNa Culture Collection (Beijing, China). The normal human colon mucosal epithelial cell line (NCM460) and seven CRC cell lines (SW620, SW480, DLD1, HCT116, KM12, LoVo, and HT-29) were grown in Dulbecco's modified Eagle's medium (Invitrogen; Thermo Fisher Scientific, Carlsbad, CA, USA) supplemented with 10\% fetal bovine serum (Invitrogen; Thermo Fisher Scientific) and 100 units of penicillin-streptomycin.

Patient information and tissue specimens. This study was conducted on a total of 125 paraffin-embedded and archived CRC samples, which were diagnosed histopathologically at the Affiliated Shenzhen Sixth Hospital of Guangdong Medical University from 2003 to 2012. Informed patient consent and approval from the Institutional Research Ethics Committee of the Affiliated Shenzhen Sixth Hospital of Guangdong Medical University was obtained for use of these clinical materials for research purposes. Clinical information regarding the samples is summarized in Table I. Ten CRC tissue samples and their matched adjacent non-cancerous colorectal tissues were frozen and stored in liquid nitrogen until further use.

Plasmids and transfection. The 3'-UTR regions of human TRAF5 (from 1801 to $2211 \mathrm{nt}$, containing a predicted conserved
miR-873 binding site) and TAB1 (from 3288 to $3700 \mathrm{nt}$, containing a predicted conserved miR-873 binding site) were generated by PCR and cloned into the modified pGL3-control luciferase reporter plasmid (Promega Corporation, Madison, WI, USA). Primer sequences were as follows: TRAF5-3'UTR sense, 5'-ACTCCGCGGATCCCAGATGATTAAATT-3' and antisense, 5'-CTAACTGCAGTTCCTTGTTCTGGGAT CAC-3'; TAB1-3'UTR sense, 5'-ACTCCGCGGCGGAGGTC CTGGCCCTCAG-3' and antisense, 5'-CTAACTGCAGCC CATGGAGGAAACAACAGGGAG-3'. Point mutations in the putative miR-873-binding seed regions of the TRAF5-3'-UTR and TAB1-3'-UTR constructs were created using a Stratagene QuikChange Mutagenesis kit (Stratagene; Agilent Technologies Inc., La Jolla, CA, USA). A miR-873 mimic, miR-873 inhibitor, and the negative control (NC) were purchased from Guangzhou RiboBio Co., Ltd. (Guangzhou, China). The sequences were as follows: miR-873 mimic, 5' GCAGGAACUUGUGAGUCU CCU-3'; miR-873-NC sense, 5'-UUUGUACUACACAAAAG UACUG-3'; miR-873 inhibitor, 5'-AGGAGACUCACAAGU UCCUGC-3'. The miR-873 mimic or miR-873 inhibitor (the miR-873 inhibitor is a locked nucleic acid (LNA)/O-Methyl oligo $(O \mathrm{Me})$ modified antisense oligonucleotide designed specifically to bind to and inhibit the endogenous miR-873 molecule) was transfected into cells using the Lipofectamine 2000 reagent (Invitrogen; Thermo Fisher Scientific) according to the manufacturer's instructions.

$R N A$ extraction, reverse transcription $(R T)$, and quantitative $P C R(q P C R)$. Total RNA from the indicated tissues or cells was extracted using the TRIzol reagent (Life Technologies; Thermo Fisher Scientific, Carlsbad, CA, USA), according to the manufacturer's instructions. Complementary DNA (cDNA) was amplified and quantified on an ABI PRISM 7500 Sequence Detection system (Applied Biosystems; Thermo Fisher Scientific, Foster City, CA, USA) using SYBR Green I (Roche Diagnostics, Grenzach-Wyhlen, Germany). miRNA quantification was determined using Bulge-loop ${ }^{\mathrm{TM}}$ miRNA quantitative reverse transcription PCR (qRT-PCR) Primer Set (one RT primer and a pair of qPCR primers for each set) specific for U6 and miR-873 that were designed and synthesized by Guangzhou RiboBio Co., Ltd. The catalog numbers of these primers were as follows: miR-873 RT primer (ssD809230648), miR-873 forward primer (ssD090525061), miR-873 reverse primer (ssD089261711), U6 RT primer (ssD0904071008), U6 forward primer (ssD0904071006), and U6 reverse primer (ss D0904071007). The quantitative PCR (qPCR) conditions were as follows: incubation at $50^{\circ} \mathrm{C}$ for $2 \mathrm{~min}, 95^{\circ} \mathrm{C}$ for $10 \mathrm{~min}$, followed by 40 cycles at $95^{\circ} \mathrm{C}$ for $15 \mathrm{sec}$, and $60^{\circ} \mathrm{C}$ for $1 \mathrm{~min}$. The expression of miR-873 was defined based on the quantification cycle $(\mathrm{Cq})$, and the relative fold changes between normal human colon mucosal epithelial cell line and the CRC cell lines, and between CRC tissues and their tumor-adjacent tissues (TATs), were calculated according to the formula $2^{-[(C q \text { of miR-873) - (Cq of } U 6)]}$ after normalization to the expression of U6 small nuclear RNA as a reference (30). REST-MCS beta software version 2 was used to further analyze the qPCR data.

MTT assays. Cells $\left(2 \times 10^{3} /\right.$ well) were seeded into 96-well plates. Transfection was performed $12 \mathrm{~h}$ later. At the indicated time-points, $100 \mu 1$ of sterile 3-(4,5-Dimethyl-2-thiazolyl)-2, 
Table I. Clinicopathological characteristics of studied patients and expression of mir-873 in CRC.

\begin{tabular}{|c|c|c|}
\hline Factors & No. & $(\%)$ \\
\hline \multicolumn{3}{|l|}{ Sex } \\
\hline Male & 68 & 54.4 \\
\hline Female & 57 & 45.6 \\
\hline \multicolumn{3}{|c|}{ Age (years) } \\
\hline$\leq 62$ & 64 & 51.2 \\
\hline$>62$ & 61 & 48.8 \\
\hline \multicolumn{3}{|l|}{ Tumor site } \\
\hline Colon & 61 & 48.8 \\
\hline Rectal & 64 & 51.2 \\
\hline \multicolumn{3}{|c|}{ Dukes' stage } \\
\hline A & 17 & 13.6 \\
\hline B & 36 & 28.8 \\
\hline $\mathrm{C}$ & 45 & 36.0 \\
\hline $\mathrm{D}$ & 27 & 21.6 \\
\hline \multicolumn{3}{|c|}{ Clinical stage } \\
\hline I & 17 & 13.6 \\
\hline II & 37 & 29.6 \\
\hline III & 44 & 35.2 \\
\hline IV & 27 & 21.6 \\
\hline \multicolumn{3}{|c|}{$\mathrm{T}$ classification } \\
\hline $\mathrm{T}_{1}$ & 2 & 1.6 \\
\hline $\mathrm{T}_{2}$ & 30 & 24.0 \\
\hline $\mathrm{T}_{3}$ & 42 & 33.6 \\
\hline $\mathrm{T}_{4}$ & 51 & 40.8 \\
\hline \multicolumn{3}{|c|}{$\mathrm{N}$ classification } \\
\hline $\mathrm{N}_{0}$ & 63 & 50.4 \\
\hline $\mathrm{N}_{1}$ & 39 & 31.2 \\
\hline $\mathrm{N}_{2}$ & 23 & 18.4 \\
\hline \multicolumn{3}{|c|}{ M classification } \\
\hline $\mathrm{M}_{0}$ & 97 & 77.6 \\
\hline $\mathrm{M}_{1}$ & 28 & 22.4 \\
\hline \multicolumn{3}{|c|}{ Histological differentiation } \\
\hline Well & 29 & 23.2 \\
\hline Moderate & 65 & 52.0 \\
\hline Poor & 31 & 24.8 \\
\hline \multicolumn{3}{|l|}{ Vital status } \\
\hline Alive & 49 & 39.2 \\
\hline Dead & 76 & 60.8 \\
\hline \multicolumn{3}{|c|}{ Expression of mir-873 } \\
\hline Low & 62 & 49.6 \\
\hline High & 63 & 50.4 \\
\hline
\end{tabular}

CRC, colorectal cancer; T, tumor; $\mathrm{N}$, node; $\mathrm{M}$, metastasis.

5-diphenyl-2H-tetrazoliumbromide (MTT) dye $(0.5 \mathrm{mg} / \mathrm{ml}$; Sigma-Aldrich, St. Louis, MI, USA) was added and incubated for $4 \mathrm{~h}$ at $37^{\circ} \mathrm{C}$. The culture medium was subsequently removed, and $150 \mu 1$ of dimethyl sulfoxide (DMSO; Sigma-Aldrich) was added. The absorbance was assessed at a wavelength of $490 \mathrm{~nm}$. All experiments were performed in triplicate.
Colony formation assay. Cells $\left(8 \times 10^{2} /\right.$ well) were seeded into 6 -well plates and cultured for 10 days. The colonies were stained with $1 \%$ crystal violet for $30 \mathrm{sec}$ after incubation with $10 \%$ formaldehyde for $5 \mathrm{~min}$. Colonies were counted only if they contained more than 50 cells, according to the established criteria for colony formation (31-33).

Western blot analysis. Cell lysates were separated by $10 \%$ sodium dodecyl sulfate-polyacrylamide gel electrophoresis (SDS-PAGE) and transferred to polyvinylidene fluoride (PVDF) membranes (EMD Millipore, Billerica, MA, USA). The membranes were probed with antibodies against TRAF5 (anti-TRAF5 antibody; 1:1,000; mouse, polyclonal; cat. no. SAB1409766) and TAB1 (anti-TAB1 antibody; 1:500; rabbit, polyclonal; cat. no. SAB4301002; both from Sigma-Aldrich) overnight at $4^{\circ} \mathrm{C}$, followed by incubation with horseradish peroxidase-conjugated secondary antibodies (1:2,000; goat; cat. no. 7074 and 1:2,000; horse; cat. no. 7076; Cell Signaling Technology, Danvers, MA, USA) for $1 \mathrm{~h}$ at $20^{\circ} \mathrm{C}$. The membranes were stripped and re-probed with an anti- $\alpha$-tubulin antibody (Sigma-Aldrich) as a loading control.

Luciferase assay. Cells ( $2 \times 10^{4} /$ well) were seeded in triplicate in 48 -well plates and allowed to settle for $24 \mathrm{~h}$. Next, $100 \mathrm{ng}$ of the luciferase reporter plasmids or the control plasmid, both with $1 \mathrm{ng}$ of pRL-TK Renilla plasmid (Promega Corporation), were transfected into cells using the Lipofectamine 2000 reagent (Invitrogen; Thermo Fisher Scientific), according to the manufacturer's recommendation. Luciferase and Renilla signals were assessed $24 \mathrm{~h}$ after transfection using a Dual Luciferase Reporter assay kit (Promega Corporation), according to the manufacturer's instructions.

Immunohistochemistry (IHC) and hematoxylin and eosin $(H \& E)$ staining. Histology was performed to quantify Ki67 expression in 10 paraffin-embedded human CRC samples. IHC was performed on sections using an anti-Ki67 antibody (1:1; mouse, monoclonal; cat. no. IR62661-2; Dako; Agilent Technologies, Inc., Glostrup, Denmark). H\&E staining was performed using Mayer's hematoxylin solution. Immunostaining of the sections was quantified and scored independently by two observers based on both the proportion of positively-stained tumor cells and the intensity of staining. The proportion of tumor cells enriched for Ki67 was scored as follows: 0 (no positive tumor cells), $1(<10 \%$ positive tumor cells), 2 (10-50\% positive tumor cells), and 3 ( $>50 \%$ positive tumor cells). The intensity of staining was scored according to the following criteria: 0 (no staining), 1 (weak staining, light yellow), 2 (moderate staining, yellow brown), and 3 (strong staining, brown). The staining index (SI) was calculated as the proportion of positive tumor cells $x$ the staining intensity score. Using this scoring method, we evaluated the expression of Ki67 by determining the SI, which was scored as $0,1,2,3,4,6$ and 9 .

Statistical analysis. All values are presented as the means \pm standard deviation (SD). Student's t-test was used to determine the statistical differences. The Chi-square test was used to analyze the relationship between miR-873 expression and clinicopathological characteristics. Multivariate statistical analysis was performed using a Cox regression model. Survival curves 


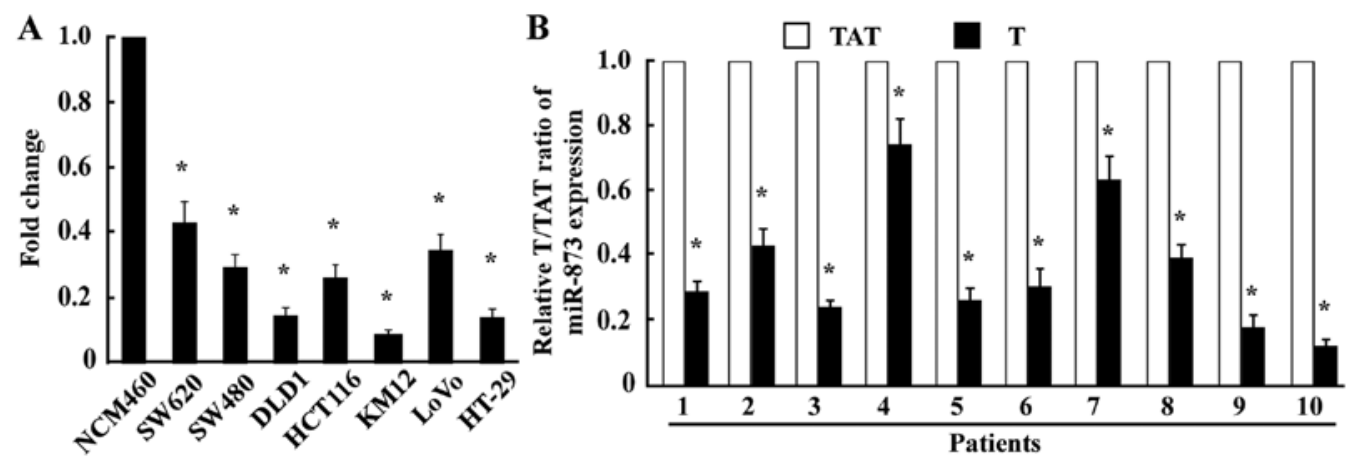

Figure 1. miR-873 expression is reduced in CRC cell lines and CRC tissues. (A) qPCR analysis of miR-873 expression in normal colon mucosal epithelial cell line NCM460 and seven cultured CRC cell lines (SW620, SW480, DLD1, HCT116, KM12, LoVo, and HT-29). (B) qPCR analysis of miR-873 expression in ten pairs of CRC samples (T) and TATs. Transcript levels were normalized to U6 expression. ${ }^{*} \mathrm{P}<0.05$. CRC, colorectal cancer; qPCR, quantitative PCR; TATs, tumor-adjacent tissues.
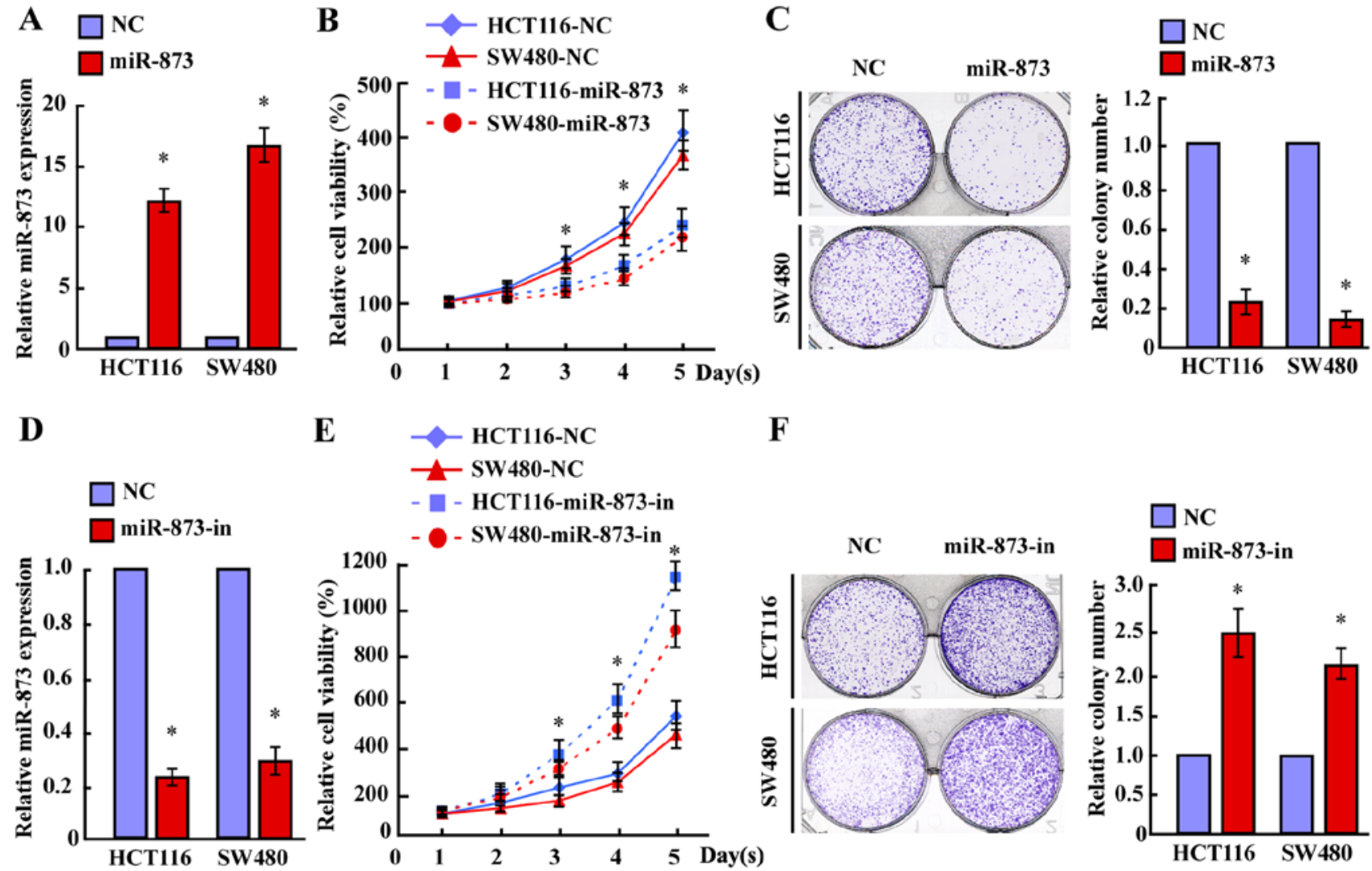

Figure 2. miR-873 inhibits proliferation of CRC cells. (A and D) The expression of miR-873 in HCT116 and SW480 cells transfected with (A) miR-873 mimic (miR-873) or (D) miR-873 inhibitor (miR-873-in) as assessed by qPCR. (B and E) The effects of miR-873 overexpression on the growth of CRC cells HCT116 and SW480. MTT assays revealed that (B) miR-873 reduced cell proliferation, whereas (E) miR-873 inhibitor-transfected HCT116 and SW480 cells proliferated more rapidly than the control cells. (C and F) Representative images (left) and quantification (right) of colonies for the indicated cells, as determined by a colony formation assay. ${ }^{\mathrm{P}}<0.05$. CRC, colorectal cancer; qPCR, quantitative PCR.

were plotted using the Kaplan-Meier method and compared using the log-rank test. Statistical analyses were performed using the SPSS 19.0 software (SPSS, Chicago, IL, USA). P $\leq 0.05$ was considered to indicate a statistically significant result.

\section{Results}

miR-873 is downregulated in CRC cell lines and CRC tissues. To examine the expression levels of miR-873 expression in $\mathrm{CRC}$, we conducted qPCR in one normal human colon mucosal epithelial cell line, seven CRC cell lines, and ten pairs of CRC tissues and their TATs. The results revealed that miR-873 was markedly decreased in all seven CRC cell lines (SW620, SW480, DLD1, HCT116, KM12, LoVo, and HT-29) compared with the normal human colon mucosal epithelial cell line NCM460 (Fig. 1A). Consistent with the results obtained from the cell lines, miR-873 expression in the ten CRC tissue samples was significantly lower compared with that in their TATs (Fig. 1B), indicating that the expression of miR-873 was downregulated in CRC. 
A

B

C

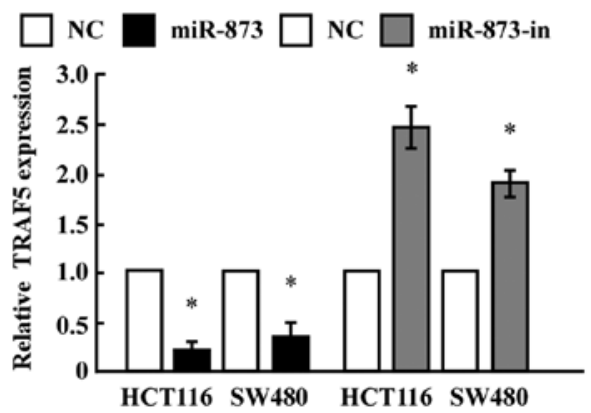

$\mathbf{E}$

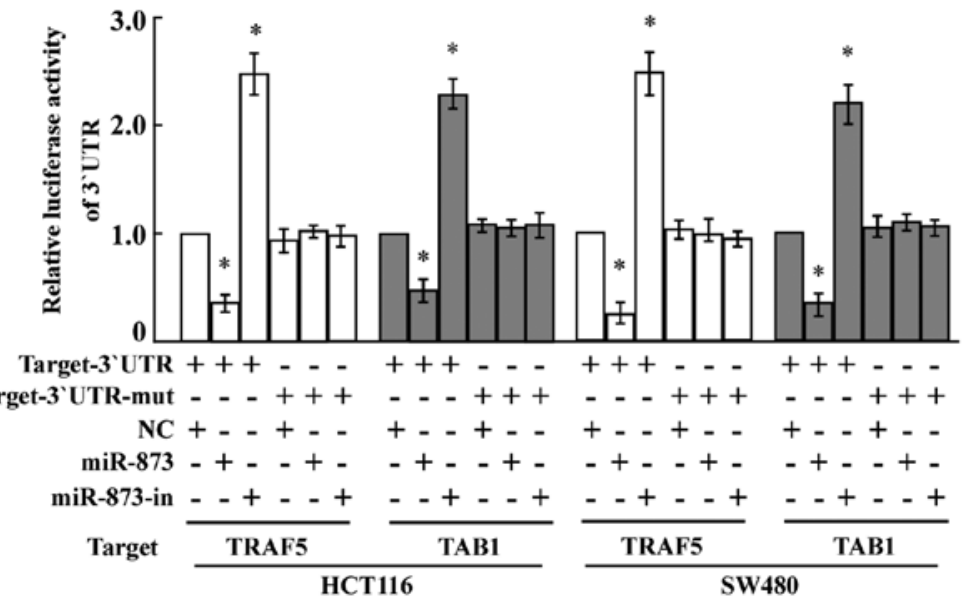

TRAF5-3'UTR

miR-873

TRAF5-3 UTR-mut

TAB1-3'UTR

miR-873

TAB1-3'UTR-mut

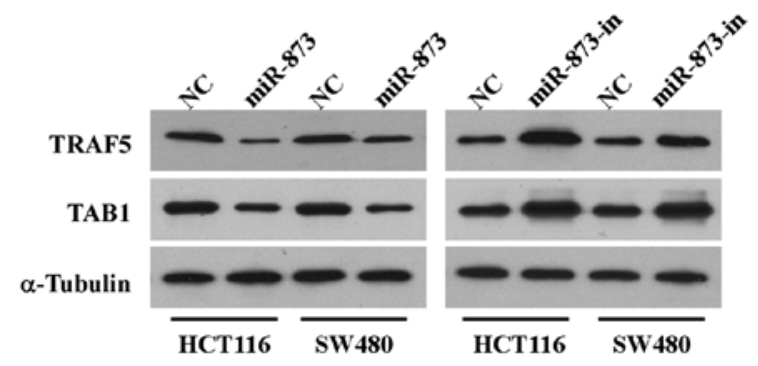

D

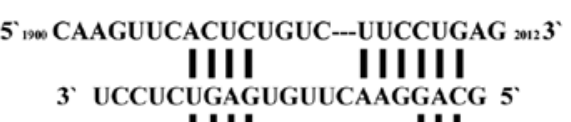

IIII

III

5 ' 1900 CAAGUUCACUCUGUC---UUCGACAG 20123

\section{IIIIIII}

3. UCCUCUGAGUGUUCAAGGACG 5

III
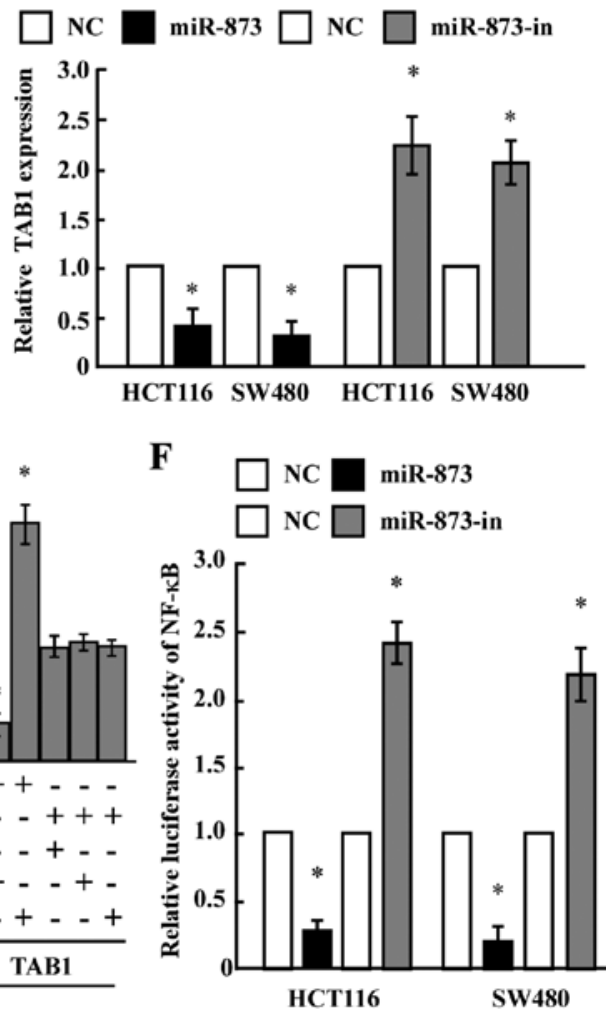

Figure 3. miR-873 directly targets TRAF5 and TAB1 to inhibit NF- $\mathrm{kB}$ signaling. (A) Predicted binding sites of miR-873 to the 3'-UTRs of TRAF5 and TAB1. (B-D) Western blotting and quantification of TRAF5 and TAB1 in the indicated cells. $\alpha$-tubulin was used as a loading control. The relative expression of the proteins was quantified by Image-Pro 6.0 software. (E) Luciferase assays on HCT116 and SW480 cells transfected with the pGL3 control reporter, pGL3-target-3'-UTR reporter, or pGL3-target-3'-UTR-mut reporter with miR-873 mimic or miR-873 inhibitor oligonucleotides. (F) NF-kB luciferase reporter activity in the indicated cells. Error bars represent the mean $\pm \mathrm{SD}$ from three independent experiments. ${ }^{*} \mathrm{P}<0.05$. TRAF5, tumor necrosis receptor-associated factor 5; TAB1, TGF- $\beta$ activated kinase 1 (MAP3K7) binding protein 1 ; NF- $\kappa B$, nuclear factor $\kappa \mathrm{B}$.

miR-873 inhibits the proliferation of CRC cells. To investigate the biological significance of miR-873 downregulation during the progression of CRC, we transfected the miR- 873 mimic into HCT116 and SW480 cell lines. MTT and colony formation assays revealed that overexpression of miR-873 markedly decreased the growth rates of both CRC lines compared with the negative control (NC) (Fig. 2A-C). Furthermore, transfection of the miR-873 inhibitor significantly increased the growth rates of both CRC lines compared with that of the NC (Fig. 2D-F). However, neither transfection of miR-873 nor the miR-873 inhibitor altered the apoptotic rates of CRC cells (data not shown). Therefore, these results revealed that miR-873 suppressed the proliferation of CRC cells.

TRAF5 and TAB1 are direct targets of miR-873 in CRC cells. To identify the direct targets of miR-873 regulation, we searched publicly available databases (TargetScan, Pictar, and miRANDA) and found that TRAF5 and TAB1, which encode components of the NF- $\kappa \mathrm{B}$ pathway, may be potential targets (Fig. 3A). As predicted, western blot analysis revealed that 
Table II. Correlation between clinicopathological features of CRC patients and expression of mir-873.

mir-873 expression

Patient characteristics Low or none (\%) High (\%) P-value

\section{Sex}

Male

Female

$31(24.8)$

31 (24.8)

37 (29.6)

0.372

Age (years)

$\leq 62$

$>62$

31 (24.8)

31 (24.8)

33 (26.4)

$30(24.0)$

0.859

Dukes' stage

A
B
C
D

$6 \quad(4.8)$

$11 \quad(8.8)$

$11(8.8)$

$25(20.0)$

$26(20.8)$

19 (15.2)

19 (15.2)

8 (6.4)

Clinical stage

I

II

III

IV

$6(4.80)$

$11(8.80)$

$26(20.8)$

19 (15.2)

$25(20.0)$

20 (16.0)

7 (5.6)

T classification

\section{T1}

T2

$\begin{array}{ll}0 & (0.0)\end{array}$

16 (12.8)

2 (1.6)

14 (11.2)

15 (12.0)

27 (21.6)

T3

$31(24.8)$

$20(16.0)$

$\mathrm{N}$ classification

$\begin{array}{llll}\mathrm{N}_{0} & 23(18.4) & 40(32.0) & 0.001 \\ \mathrm{~N}_{1} & 20(16.0) & 19(15.2) & \\ \mathrm{N}_{2} & 19(15.2) & 4(3.2) & \\ \mathrm{M} \text { classification } & & & \\ \mathrm{M}_{0} & 42(33.6) & 55(44.0) & 0.010 \\ \mathrm{M}_{1} & 20(16.0) & 8(6.4) & \end{array}$

Histological

differentiation

Well
Moderate
Poor

$21(16.8)$

$26(20.8)$

15 (12.0)

$\begin{array}{lll}8 & (6.4) \quad 0.014\end{array}$

39 (31.2)

16 (12.8)

Vital status

Alive

15 (12.0)

34 (27.2)

0.001

Dead

47 (37.6)

29 (23.2)

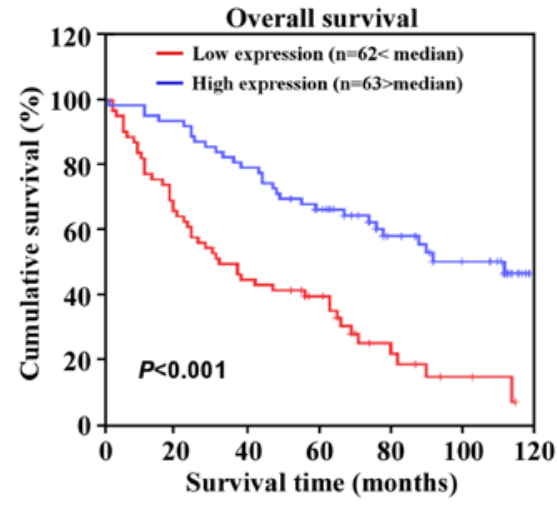

Figure 4. Expression of miR-873 is correlated with CRC prognosis. Kaplan-Meier analysis of the correlation between the miR-873 expression levels and the overall survival rates of patients with CRC with high $(>$ the median, $n=63$ ) and low ( $<$ the median, $n=62$ ) miR-873 expression.

we subcloned the 3'-UTRs of TRAF5 and TAB1 into the pGL3 luciferase reporter. Transfection of miR-873 consistently attenuated the luciferase activity of the TRAF5-3'-UTR and TAB1-3'-UTR luciferase reporter in both HCT116 and SW480 cells, whereas transfection of the miR-873 inhibitor rescued luciferase suppression. However, dysregulation of miR-873 did not result in the alteration of the reporter activities driven by the 3'-UTRs of TRAF5 and TAB1 mutated within the miR-873-binding seed regions (Fig. 3E). Collectively, these results further supported the view that TRAF5 and TAB1 are genuine targets of miR-873.

Furthermore, the luciferase assay revealed that ectopic expression of miR-873 in HCT116 and SW480 cells significantly reduced $\mathrm{NF}-\kappa \mathrm{B}$ luciferase activity, while ectopic expression of the miR-873 inhibitor enhanced NF- $\kappa \mathrm{B}$ luciferase activity (Fig. 3F). This suggested an important role for $\mathrm{NF}-\kappa \mathrm{B}$ signaling in the CRC cell proliferation induced by miR-873 downregulation.

miR-873 expression is correlated with clinical features and prognosis of patients with CRC. To further evaluate whether miR-873 downregulation was associated with the clinical features or prognosis of CRC, we examined the expression of miR-873 in a large cohort of clinical CRC samples using qPCR and performed a correlation analysis between the clinicopathological features and the expression of miR-873. The data revealed that miR-873 expression was inversely correlated with Dukes' stage $(\mathrm{P}=0.005)$, clinical stage $(\mathrm{P}=0.002)$, tumor-node-metastasis (TNM) classification $(\mathrm{T}, \mathrm{P}=0.036$; $\mathrm{N}, \mathrm{P}=0.001 ; \mathrm{M}, \mathrm{P}=0.010)$, and histological differentiation $(\mathrm{P}=0.014)$ (Table II). Additionally, Kaplan-Meier survival analysis revealed that patients with CRC with lower miR-873 expression levels had shorter overall survival (Fig. 4). Moreover, univariate and multivariate analysis indicated that miR-873 expression levels were an independent prognostic factor for CRC (Table III). Collectively, these results indicated a possible link between miR-873 downregulation and CRC progression.

Clinical relevance of $\mathrm{miR}-873$ downregulation and cell proliferation in $C R C$. Finally, we examined whether the reduction in $\mathrm{miR}-873$ expression induced cell proliferation in CRC suggesting that miR-873 negatively regulated the expression of these proteins at the translation level. To further test this, ectopic expression of miR-873 in HCT116 and SW480 cells decreased the levels of the TRAF5 and TAB1 proteins, whereas ectopic expression of the miR-873 inhibitor increased their levels (Fig. 3B-D). However, we determined that the mRNA expression levels of TRAF5 and TABI did not exhibit evident alterations in the miR-873 dysregulated cells (data not shown), 
Table III. Univariate and multivariate analysis of different prognostic parameters in patients with CRC by Cox-regression analysis.

\begin{tabular}{|c|c|c|c|c|c|c|}
\hline \multirow[b]{2}{*}{ Parameters } & \multicolumn{3}{|c|}{ Univariate analysis } & \multicolumn{3}{|c|}{ Multivariate analysis } \\
\hline & $\begin{array}{l}\text { No. of } \\
\text { patients }\end{array}$ & P-value & $\begin{array}{c}\text { Regression } \\
\text { coefficient (SE) }\end{array}$ & P-value & $\begin{array}{l}\text { Relative } \\
\text { risk }\end{array}$ & $\begin{array}{c}95 \% \text { confidence } \\
\text { interval }\end{array}$ \\
\hline $\mathrm{N}$ classification & & $<0.001$ & 0.150 & $<0.001$ & 1.746 & $1.306-2.336$ \\
\hline $\mathrm{N}_{0}$ & 63 & & & & & \\
\hline $\mathrm{N}_{1}$ & 39 & & & & & \\
\hline $\mathrm{N}_{2}$ & 23 & & & & & \\
\hline M classification & & $<0.001$ & 0.260 & 0.001 & 2.405 & $1.430-4.044$ \\
\hline $\mathrm{M}_{0}$ & 97 & & & & & \\
\hline $\mathrm{M}_{1}$ & 28 & & & & & \\
\hline Expression of mir-873 & & $<0.001$ & 0.242 & 0.001 & 0.450 & $0.275-0.734$ \\
\hline Low & 62 & & & & & \\
\hline High & 63 & & & & & \\
\hline
\end{tabular}

CRC, colorectal cancer; $\mathrm{N}$, node; $\mathrm{M}$, metastasis.
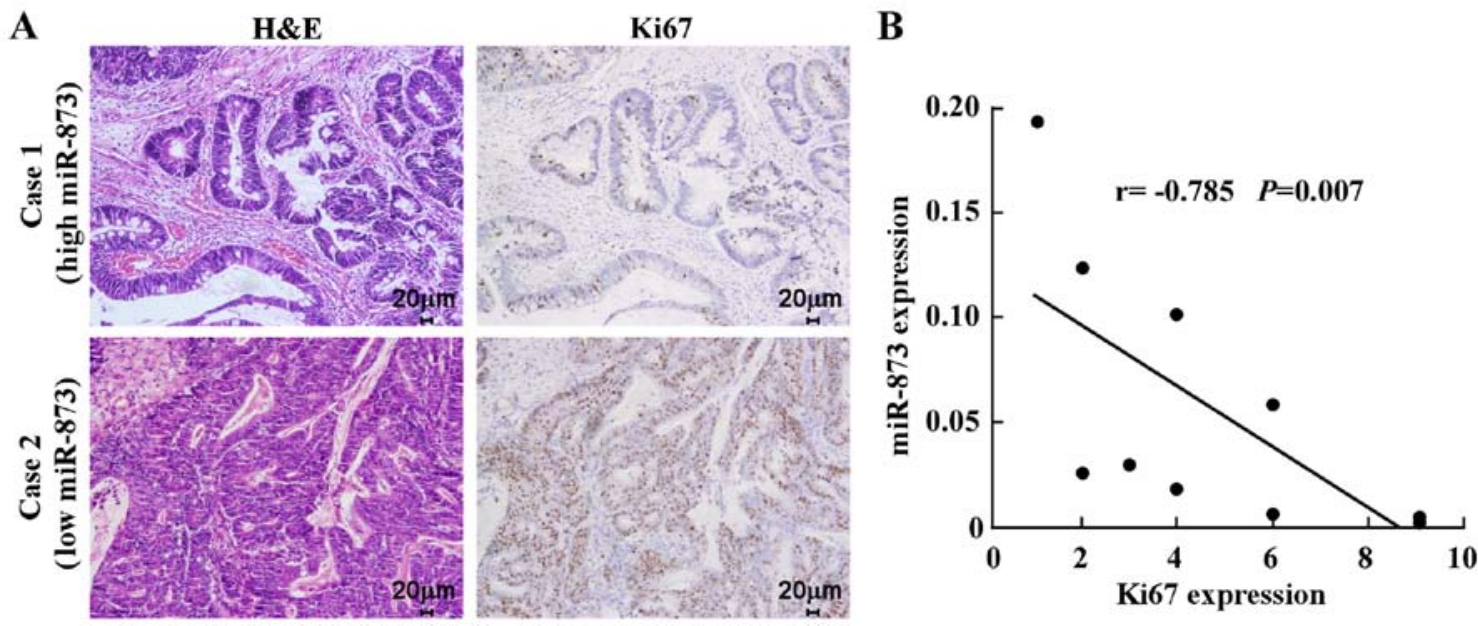

Figure 5. Clinical relevance of miR-873 downregulation and cell proliferation in CRC. (A) miR-873 levels were inversely correlated with Ki67 in ten primary CRC specimens. Two representative specimens for low and high levels of miR-873 are displayed. Original magnification, x200. (B) Analysis of the correlation between miR-873 and Ki67 expression in ten CRC specimens. The relative data of qPCR were used as the ordinate and the staining index of Ki67 as the abscissa. CRC, colorectal cancer; qPCR, quantitative PCR.

samples and whether this was clinically relevant. IHC analysis of ten CRC specimens revealed that low miR-873-expressed specimens had a higher proportion of cells expressing the proliferation marker Ki67. In contrast, high miR-873-expressed specimens displayed a small proportion of Ki67-positive cells among the ten CRC specimens (Fig. 5A). Correlation analysis revealed that miR-873 expression levels were inversely correlated with Ki67 in these CRC samples (Fig. 5B). Collectively, these results established that miR-873 suppressed CRC cell proliferation via inhibition of TRAF5 and TAB1, which are key components of the NF- $\kappa \mathrm{B}$ signaling pathway.

\section{Discussion}

The key finding of the current study is that miR-873 is a tumorsuppressive miRNA in CRC. Our data revealed that miR-873 was significantly downregulated in both CRC cell lines and primary CRC specimens. Furthermore, downregulation of miR-873 expression was associated with more advanced tumor stages and poor prognoses for patients with CRC. Ectopic expression of miR-873 inhibited CRC cell proliferation, whereas silencing of miR-873 promoted cell proliferation. In addition, we demonstrated that miR-873 suppressed the NF- $\mathrm{KB}$ pathway by directly targeting TRAF5 and $T A B 1$, which encode vital components of this pathway. Collectively, our results demonstrated that miR-873 plays a critical role in the tumorigenesis and progression of CRC and may represent an important target for clinical intervention of CRC.

Previous research has revealed that miRNAs are involved in tumor initiation, progression, metastasis, and response to chemotherapy in CRC. In 2003, the first study describing the roles of miRNAs in CRC was published, which revealed 
that miR-143 and miR-145 were specifically dysregulated in $\mathrm{CRC}$, and multiple other miRNAs were found to contribute to CRC by regulating critical target mRNAs (34). For example, several studies have revealed the dysregulation of a variety of tissue-specific miRNAs, e.g., miR-21, miR-181b, miR-155, miR-92a, and let-7, as well as some circulating miRNAs, e.g., miR-26a, miR-21, miR-126, and miR-203 in CRC $(35,36)$. These miRNAs exert their effects by negatively regulating their targets, such as p53, c-Met, K-Ras, COX-2, Rb, and the Bcl-2 family (37). Accumulating evidence indicates that miRNAs may serve as targets for miRNA-based therapeutics of CRC. Inhibition of overexpressed oncogenic miRNAs or introduction of tumor-suppressive miRNAs into cancer cells may represent novel treatment strategies for CRC therapy in the future (38).

Previously, it was shown that miR-873 is dysregulated in a variety of malignancies. Notably, miR-873 was downregulated in glioblastoma, and inhibited tumorigenesis and metastasis by suppressing the expression of insulin-like growth factor 2 mRNA-binding protein 1 (IGF2BP1) $(24,25)$. In addition, miR-873 enhanced the sensitivity of glioma cells to cisplatin by targeting $\mathrm{Bcl}-2$ (26). It was also reported that overexpression of miR-873 increased the sensitivity of ovarian cancer cells to cisplatin and paclitaxel by targeting multidrug resistance protein 1 (MDR1) (27). However, there are also studies which have reported that cisplatin is not a $\mathrm{P}$-glycoprotein substrate and multidrug resistance induced by cisplatin in ovarian carcinoma cell lines was not due to overexpression of MDR1 and MDR3, both of which are P-glycoproteins $(39,40)$. Therefore, the controversial mechanisms of multidrug resistance induced by cisplatin in ovarian carcinoma warrant further investigation. In addition, miR-873 was downregulated in tamoxifen-resistant breast cancer cell lines, while overexpression of miR-873 reversed tamoxifen resistance by targeting cyclin-dependent kinase 3 (CDK3) (28). By contrast, however, Gao et al reported that miR-873 may act as an oncogene in lung adenocarcinoma since it increased tumor cell proliferation and migration via direct inhibition of SRCIN1 expression (29). Collectively, these findings indicated that miR-873 can act as either a tumor-suppressive or -promoting miRNA depending on the type of cancer. In this context, we demonstrated that miR-873 inhibited CRC cell proliferation and functioned as a tumorsuppressive miRNA in CRC. Meanwhile, the inversed clinical relevance of miR-873 reduction with higher Ki67 signaling further supported the suppressive effect of miR-873 on proliferation in CRC. However, the inhibitory effect of miR-873 on proliferation in CRC warrants further investigation using an in vivo mouse model. In addition, the expression and biological function of miR-873 in other gastrointestinal tract cancers also warrant further clarification.

$\mathrm{NF}-\kappa \mathrm{B}$ is a family of transcription factors that controls the expression of a large number of genes related to inflammation, immune responses, development, survival, and proliferation (41). Since its discovery nearly three decades ago (42), numerous studies have reported that the $\mathrm{NF}-\kappa \mathrm{B}$ signaling pathway is frequently activated in a variety of human cancers and it is associated with tumor initiation and progression $(6,8)$. The NF- $\mathrm{NB}$ signaling pathway plays critical roles in the physiological and pathological processes of $\mathrm{CRC}$, and the relationship between $\mathrm{CRC}$ development and $\mathrm{NF}-\kappa \mathrm{B}$ signaling is becoming clear (43). Multiple research groups revealed that the constitutively activated form of $\mathrm{NF}-\kappa \mathrm{B}$ was frequently expressed in CRC cells (44-46). NF- $\kappa \mathrm{B}$ may contribute to the progression of $\mathrm{CRC}$ by regulating the expression of diverse target genes that are involved in cell proliferation, angiogen-

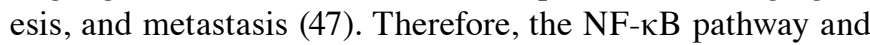
its upstream and downstream network constitute a potential druggable target for therapeutic interventions (48). Although IKK complex-mediated NF- $\kappa \mathrm{B}$ activation has been studied in great detail, the regulatory mechanism of the constitutive activation of $\mathrm{NF}-\kappa \mathrm{B}$ in $\mathrm{CRC}$ remains largely unknown. Herein, we found that miR-873 significantly inhibited the $\mathrm{NF}-\kappa \mathrm{B}$ pathway by directly targeting TRAF5 and TAB1, key components of the NF- $\kappa$ B pathway. Thus, our results indicated that miR-873 plays a regulatory role in $\mathrm{NF}-\kappa \mathrm{B}$ activation, and the effect of miR-873-induced activation of NF- $\mathrm{NB}$ on invasion, angiogenesis, or metastasis of CRC warrant further investigation.

In conclusion, the present study reported that miR-873 was downregulated in CRC and the expression level of miR-873 was correlated with CRC progression and prognosis. We have demonstrated, for the first time, that the upregulation of miR-873 markedly inhibited CRC cell proliferation by inhibiting the expression of two key components (TRAF5 and TAB1) of the $\mathrm{NF}-\kappa \mathrm{B}$ pathway. Therefore, our results demonstrated that miR-873 may play an important role in the progression of CRC and could represent a potential therapeutic target for CRC.

\section{Acknowledgements}

This study was supported by the Natural Science Foundation of China (nos. 81402310, 81672957 and 91529301) and the Science and Technology Innovation Committee of Shenzhen Municipality (nos. JCYJ20140411093600199 and JCYJ20160428180814307).

\section{References}

1. Haggar FA and Boushey RP: Colorectal cancer epidemiology: Incidence, mortality, survival, and risk factors. Clin Colon Rectal Surg 22: 191-197, 2009.

2. Weng W, Wei Q, Toden S, Yoshida K, Nagasaka T, Fujiwara T, Cai S, Qin H, Ma Y and Goel A: Circular RNA ciRS-7-a promising prognostic biomarker and a potential therapeutic target in colorectal cancer. Clin Cancer Res 23: 3918-3928, 2017.

3. Zeng J, Tang ZH, Liu S and Guo SS: Clinicopathological significance of overexpression of interleukin- 6 in colorectal cancer. World J Gastroenterol 23: 1780-1786, 2017.

4. Deng J, Lei W, Fu JC, Zhang L, Li JH and Xiong JP: Targeting miR-21 enhances the sensitivity of human colon cancer HT-29 cells to chemoradiotherapy in vitro. Biochem Biophys Res Commun 443: 789-795, 2014.

5. Wieser M, Sauerland S, Arnold D, Schmiegel W and ReinacherSchick A: Peri-operative chemotherapy for the treatment of resectable liver metastases from colorectal cancer: A systematic review and meta-analysis of randomized trials. BMC Cancer 10: 309-321, 2010.

6. Moghimi-Dehkordi B and Safaee A: An overview of colorectal cancer survival rates and prognosis in Asia. World J Gastrointest Oncol 4: 71-75, 2012.

7. Karin $M$ and Greten FR: NF-kappaB: Linking inflammation and immunity to cancer development and progression. Nat Rev Immunol 5: 749-759, 2005.

8. Hayden MS and Ghosh S: Shared principles in NF-kappaB signaling. Cell 132: 344-362, 2008.

9. Hoesel B and Schmid JA: The complexity of NF- $\mathrm{kB}$ signaling in inflammation and cancer. Mol Cancer 12: 86, 2013. 
10. Wu D, Wu P, Zhao L, Huang L, Zhang Z, Zhao S and Huang J: NF- $\kappa \mathrm{B}$ expression and outcomes in solid tumors: A systematic review and meta-analysis. Medicine (Baltimore) 94: e1687, 2015.

11. Chen Z, Zhao L, Zhao F, Yang G and Wang J: MicroRNA-26b regulates cancer proliferation migration and cell cycle transition by suppressing TRAF5 in esophageal squamous cell carcinoma. Am J Transl Res 8: 1957-1970, 2016.

12. Jiang L, Yu L, Zhang X, Lei F, Wang L, Liu X, Wu S, Zhu J, Wu G, Cao L, et al: miR-892b silencing activates NF- $\mathrm{BB}$ and promotes aggressiveness in breast cancer. Cancer Res 76: 1101-1111, 2016.

13. Harhaj EW and Dixit VM: Deubiquitinases in the regulation of NF- $\kappa$ B signaling. Cell Res 21: 22-39, 2011.

14. Khella HWZ, Daniel N, Youssef L, Scorilas A, Nofech-Mozes R, Mirham L, Krylov SN, Liandeau E, Krizova A, Finelli A, et al miR-10b is a prognostic marker in clear cell renal cell carcinoma. J Clin Pathol 70: 854-859, 2017.

15. Chen J, Wang M, Guo M, Xie Y and Cong YS: miR-127 regulates cell proliferation and senescence by targeting BCL6. PLoS One 8: e80266, 2013

16. Li M, Wang Y, Song Y, Bu R, Yin B, Fei X, Guo Q and Wu B: MicroRNAs in renal cell carcinoma: A systematic review of clinical implications (Review). Oncol Rep 33: 1571-1578, 2015.

17. Zhao G, Cai C, Yang T, Qiu X, Liao B, Li W, Ji Z, Zhao J, Zhao H, Guo M, et al: MicroRNA-221 induces cell survival and cisplatin resistance through PI3K/Akt pathway in human osteosarcoma. PLoS One 8: e53906, 2013.

18. Go H, Jang JY, Kim PJ, Kim YG, Nam SJ, Paik JH, Kim TM, Heo DS, Kim CW and Jeon YK: MicroRNA-21 plays an oncogenic role by targeting FOXO1 and activating the PI3K/ AKT pathway in diffuse large B-cell lymphoma. Oncotarget 6: 15035-15049, 2015.

19. Li Y, VandenBoom TG II, Kong D, Wang Z, Ali S, Philip PA and Sarkar FH: Up-regulation of miR-200 and let-7 by natural agents leads to the reversal of epithelial-to-mesenchymal transition in gemcitabine-resistant pancreatic cancer cells. Cancer Res 69: 6704-6712, 2009.

20. Peng Y, Liu YM, Li LC, Wang LL and Wu XL: microRNA-503 inhibits gastric cancer cell growth and epithelial-to-mesenchymal transition. Oncol Lett 7: 1233-1238, 2014.

21. Nofech-Mozes R, Khella HW, Scorilas A, Youssef L, Krylov SN, Lianidou E, Sidiropoulos KG, Gabril M, Evans A and Yousef GM MicroRNA-194 is a marker for good prognosis in clear cell renal cell carcinoma. Cancer Med 5: 656-664, 2016.

22. Rapti SM, Kontos CK, Papadopoulos IN and Scorilas A: High miR-96 levels in colorectal adenocarcinoma predict poo prognosis, particularly in patients without distant metastasis at the time of initial diagnosis. Tumour Biol 37: 11815-11824, 2016

23. Lee TS, Jeon HW, Kim YB, Kim YA, Kim MA and Kang SB: Aberrant microRNA expression in endometrial carcinoma using formalin-fixed paraffin-embedded (FFPE) tissues. PLoS One 8: e81421, 2013.

24. Skalsky RL and Cullen BR: Reduced expression of brain-enriched microRNAs in glioblastomas permits targeted regulation of a cell death gene. PLoS One 6: e24248, 2011.

25. Wang RJ, Li JW, Bao BH, Wu HC, Du ZH, Su JL, Zhang MH and Liang HQ: MicroRNA-873 (miRNA-873) inhibits glioblastoma tumorigenesis and metastasis by suppressing the expression of IGF2BP1. J Biol Chem 290: 8938-8948, 2015.

26. Chen X, Zhang Y, Shi Y, Lian H, Tu H, Han S, Peng B, Liu W and He X: miR-873 acts as a novel sensitizer of glioma cells to cisplatin by targeting Bcl-2. Int J Oncol 47: 1603-1611, 2015.

27. Wu DD, Li XS, Meng XN, Yan J and Zong ZH: MicroRNA-873 mediates multidrug resistance in ovarian cancer cells by targeting ABCB1. Tumour Biol 37: 10499-10506, 2016

28. Cui J, Bi M, Overstreet AM, Yang Y, Li H, Leng Y, Qian K, Huang Q, Zhang C, Lu Z, et al: miR-873 regulates Era transcriptional activity and tamoxifen resistance via targeting CDK3 in breast cancer cells. Oncogene 22: 1-13, 2014.
29. Gao Y, Xue Q, Wang D, Du M, Zhang Y and Gao S: miR-873 induces lung adenocarcinoma cell proliferation and migration by targeting SRCIN1. Am J Transl Res 7: 2519-2526, 2015.

30. Liao WT, Ye YP, Zhang NJ, Li TT, Wang SY, Cui YM, Qi L, Wu P, Jiao HL, Xie YJ, et al: MicroRNA-30b functions as a tumour suppressor in human colorectal cancer by targeting KRAS, PIK3CD and BCL2. J Pathol 232: 415-427, 2014.

31. Miao Y, Li J, Qiu X, Li Y, Wang Z and Luan Y: miR-27a regulates the self renewal of the H446 small cell lung cancer cell line in vitro. Oncol Rep 29: 161-168, 2013.

32. Li XX, Huang LY, Peng JJ, Liang L, Shi DB, Zheng HT and Cai SJ: Klotho suppresses growth and invasion of colon cancer cells through inhibition of IGF1R-mediated PI3K/AKT pathway. Int J Oncol 45: 611-618, 2014

33. Qu L, Deng B, Zeng Y and Cao Z: Decreased expression of the Nkx2.8 gene correlates with tumor progression and a poor prognosis in HCC cancer. Cancer Cell Int 14: 28, 2014.

34. Michael MZ, O' Connor SM, van Holst Pellekaan NG, Young GP and James RJ: Reduced accumulation of specific microRNAs in colorectal neoplasia. Mol Cancer Res 1: 882-891, 2003.

35. Orang AV and Barzegari A: MicroRNAs in colorectal cancer: From diagnosis to targeted therapy. Asian Pac J Cancer Prev 15: 6989-6999, 2014.

36. Kijima T, Hazama S, Tsunedomi R, Tanaka H, Takenouchi H, Kanekiyo S, Inoue Y, Nakashima M, Iida M, Sakamoto K, et al: MicroRNA-6826 and -6875 in plasma are valuable non-invasive biomarkers that predict the efficacy of vaccine treatment against metastatic colorectal cancer. Oncol Rep 37: 23-30, 2017.

37. Wang J, Du Y, Liu X, Cho WC and Yang Y: MicroRNAs as regulator of signaling networks in metastatic colon cancer. Biomed Res Int 2015: 823620, 2015.

38. Amirkhah R, Schmitz U, Linnebacher M, Wolkenhauer O and Farazmand A: MicroRNA-mRNA interactions in colorectal cancer and their role in tumor progression. Genes Chromosomes Cancer 54: 129-141, 2015

39. Ren L, Xiao L, Hu J, Li Z and Wang Z: MDR1 and MDR3 genes and drug resistance to cisplatin of ovarian cancer cells. J Huazhong Univ Sci Technolog Med Sci 27: 721-724, 2007.

40. Stordal B, Hamon M, McEneaney V, Roche S, Gillet JP, O'Leary JJ, Gottesman M and Clynes M: Resistance to paclitaxel in a cisplatin-resistant ovarian cancer cell line is mediated by P-glycoprotein. PLoS One 7: e40717, 2012.

41. Hayden MS and Ghosh S: Signaling to NF-kappaB. Genes Dev 18: $2195-2224,2004$

42. Sen R and Baltimore D: Multiple nuclear factors interact with the immunoglobulin enhancer sequences. Cell 46: 705-716, 1986.

43. Sakamoto K and Maeda S: Targeting NF-kappaB for colorectal cancer. Expert Opin Ther Targets 14: 593-601, 2010

44. Sakamoto K, Maeda S, Hikiba Y, Nakagawa H, Hayakawa Y, Shibata W, Yanai A, Ogura K and Omata M: Constitutive NF-kappaB activation in colorectal carcinoma plays a key role in angiogenesis, promoting tumor growth. Clin Cancer Res 15: 2248-2258, 2009.

45. Voboril R and Weberova-Voborilova J: Constitutive NF-kappaB activity in colorectal cancer cells: Impact on radiation-induced NF-kappaB activity, radiosensitivity, and apoptosis. Neoplasma 53: 518-523, 2006.

46. Lind DS, Hochwald SN, Malaty J, Rekkas S, Hebig P, Mishra G, Moldawer LL, Copeland EM III and Mackay S: Nuclear factor- $\kappa \mathrm{B}$ is upregulated in colorectal cancer. Surgery 130: 363-369, 2001.

47. Wang S, Liu Z, Wang L and Zhang X: NF-kappaB signaling pathway, inflammation and colorectal cancer. Cell Mol Immunol 6: 327-334, 2009

48. Vaiopoulos AG, Athanasoula KC and Papavassiliou AG: NF- $\kappa B$ in colorectal cancer. J Mol Med (Berl) 91: 1029-1037, 2013.

(i) $(-)$ This work is licensed under a Creative Commons Attribution-NonCommercial-NoDerivatives 4.0 International (CC BY-NC-ND 4.0) License. 